\title{
BMJ Open What matters most to patients about primary healthcare: mixed-methods patient priority setting exercises within the PREFeR (PRioritiEs For Research) project
}

\author{
Louisa Edwards, ${ }^{\oplus 1,2}$ Melody Monro, ${ }^{3}$ Yaron Butterfield, ${ }^{4}$ Ravin Johl, ${ }^{4}$ \\ Kent Cadogan Loftsgard, ${ }^{4}$ Hayley Pelletier, ${ }^{4}$ Colleen McGavin, ${ }^{5}$ M Ruth Lavergne ${ }^{\oplus}$
}

To cite: Edwards L, Monro M, Butterfield $\mathrm{Y}$, et al. What matters most to patients about primary healthcare: mixed-methods patient priority setting exercises within the PREFeR (PRioritiEs For Research) project. BMJ Open 2019;9:e025954. doi:10.1136/ bmjopen-2018-025954

- Prepublication history and additional material for this paper are available online. To view these files, please visit the journal online (http://dx.doi. org/10.1136/bmjopen-2018025954).

Received 9 August 2018

Revised 5 April 2019

Accepted 3 June 2019
Check for updates

(C) Author(s) (or their employer(s)) 2019. Re-use permitted under CC BY-NC. No commercial re-use. See rights and permissions. Published by BMJ.

For numbered affiliations see end of article.

Correspondence to Dr M Ruth Lavergne; ruth_lavergne@sfu.ca

\section{ABSTRACT}

Objectives To identify patient-generated priority topics for future primary care research in British Columbia (BC), Canada within a diverse patient population.

Design Mixed-methods priority setting exercises framed by the dialogue model, using the nominal group technique (rank-ordered scoring) and province-wide online surveys capturing importance ratings of the top 10 primary healthcare topics from patients and primary care providers.

Setting BC, Canada.

Participants Topic identification was completed by 10 patient partners (7 female, 3 male) from the BC Primary Health Care Research Network Patient Advisory; online surveys were completed by 464 patients and 173 primary care providers.

Results The 10 members recruited to the patient advisory provided over 80 experiences of what stood out for them in $\mathrm{BC}$ primary care, which were grouped thematically into 18 topics, 10 of which were retained in province-wide surveys. Top-rated survey topics for both patients $(n=464)$ and providers $(n=173)$ included being unable to find a regular family doctor/other primary healthcare provider, support for living with chronic conditions, mental health resources and information sharing, including electronic medical records. However, all 10 topics were rated important, on average, by both groups.

Conclusions The current project activities demonstrate the feasibility of including patients in priority setting exercises for primary healthcare in general, rather than focusing on a condition-specific population or disease area. There was considerable overlap between patientgenerated topics and topics previously identified by other stakeholders, but patients identified two additional topics (mental health resources, improve and strengthen patient-provider communication). More similarities than differences in topic importance between patients and providers emerged in the online surveys. The project activities that follow (rapid literature reviews, multistakeholder dialogue) will highlight under-researched topics and inform the development of specific research questions.

\section{Strengths and limitations of this study}

- This is the only published patient priority setting project that we are aware of that addresses primary healthcare topics in general, rather than focusing on a particular health condition or issue.

- The project was informed by the dialogue model, which is a multistage, multistakeholder structured priority setting framework, but explicitly focused on patient perspectives within the exploration and consultation phases of primary healthcare topic generation.

- Patients identified new topics and added depth to topics previously identified in similar exercises among policy-makers or researchers, further highlighting the importance of patient engagement in priority setting.

- Generalisability of the current priority topics might be limited, given the characteristics of the patient advisory (eg, well educated, previous experience with research) and survey respondents (eg, predominantly female, White).

\section{INTRODUCTION}

Primary care is the first point of contact with healthcare for most patients, and supports a wide variety of health needs. It is not surprising, therefore, that a host of primary care research focuses on studying patient needs and gaps in care. However, patients are not frequently included in prioritising research ideas. ${ }^{1-3}$ At a basic level, this is problematic because patient, clinician and researcher priorities may differ significantly. ${ }^{4}$ At an applied level, intervention uptake and, consequently, any impact on health outcomes may suffer if the problems studied and outcomes suggested have not considered patients' perspectives, leading to research 'waste'. ${ }^{5}$ Patient prioritisation of topics for research ultimately benefits the intended end-users of primary healthcare 
directly-patients themselves—and makes better use of scarce resources.

There is growing recognition of the importance of involving patients in research priority setting. In response, there has been a proliferation of patient priority setting studies in recent years, with 70 articles from the UK, USA, Canada, the Netherlands and Australia identified between 2007 and 2017 in a rapid systematic review of public and patient engagement in prioritisation. ${ }^{6}$ Yet, most processes for engaging patients in research have focused on populations defined by a specific condition, ${ }^{6} 7$ rather than primary care more generally. While there have been exercises to identify primary care research priorities at clinical and academic conferences, ${ }^{8}$ these did not include patients. We have only identified a few recent publications that examined primary care priorities more generally-that is, in which the overall focus was not specific to a particular health condition-and that involved patients as one of the multistakeholder groups. ${ }^{9-12}$ Even so, these studies actually targeted more specific topics-how to promote healthy behaviours through primary care, ensuring care is guided by patient goals and preferences, and patient safety concerns-rather than allowing any topics relating to primary care experiences to emerge as priorities. In order to examine gaps in care that affect a large and diverse range of patients, it is important to enable patients to voice whatever primary healthcare priorities are most pressing for them.

There are a host of tools and techniques for priority setting. One recent methodological review found roughly equal numbers of studies that used published, structured frameworks and those that used newly developed tools (eg, surveys specific to the project). ${ }^{13}$ In terms of structured approaches, there are several multistage priority setting frameworks, such as the James Lind Alliance Priority Setting Partnerships ${ }^{14}$ and the dialogue model. ${ }^{15}$ The James Lind Alliance has developed important principles to guide priority setting for research with patients and caregivers, ${ }^{16}$ but this approach is anchored on treatment uncertainties and does not translate directly to questions related to primary care more generally. There does not appear to be a single, best method, ${ }^{17}$ nor is there clear agreement on whether using a combination of tools for priority setting is superior to using one technique alone. ${ }^{13}$ However, the use of a clearly defined approach is recommended, ${ }^{1318}$ and the choice of which method(s) may be best guided by the research question. ${ }^{17}$ The Cochrane Priority Setting Methods Group noted that researchers may adapt and implement the same methods in different ways depending on the context and research focus. ${ }^{19}$

Recognising that patient engagement early in the research process is important, but has been largely absent from priority setting exercises in primary care, the main aim of the PRioritiEs For Research (PREFeR) Project was to identify patient-generated priorities for primary care research in British Columbia (BC), Canada. This is consistent with Canada's Strategy for Patient-Priented Research, which supports research that engages patients as partners, focuses on patient-identified priorities and improves patient outcomes. ${ }^{20}$ This decision to focus on patient priorities was also shaped by the fact that research priorities for primary care had recently been identified in $\mathrm{BC}$, but this process included policy-makers, clinicians and researchers, and not patients. ${ }^{21}$ By focusing on topics that are of importance to patients, research is more likely to have positive impacts on policy and practice.

As noted in reviews, ${ }^{62}$ detail about methodology is often lacking in articles about priority setting, and this is essential to replicating approaches and understanding how and what works for patient engagement. The PREFeR Project was informed by the dialogue model, which is a multistage priority setting framework involving multiple stakeholders, but modified this model to focus solely on patient perspectives for initial topic generation. ${ }^{1}$ Here, we report the resulting patient-identified priorities and their importance for patients and primary care providers. We also overview the other project activities that are couched within the later phases of the dialogue model, which correspond to the secondary aims of PREFeR: to assess whether research is already being conducted on the patient priorities through rapid literature reviews and 'horizon scanning' surveys with BC researchers, and to explore where and why differences might occur in the survey results through a facilitated discussion ('dialogue') with patients, providers, researchers and policy-makers. Taken together, results from these activities are key considerations for setting future research questions.

\section{METHODS}

\section{Patient and public involvement}

The BC Primary Health Care Research Network (BC-PHCRN) Patient Advisory (hereafter, referred to as the patient advisory) was formed to conduct this priority setting exercise, as well as gather their input into multiple studies supported by the BC-PHCRN. Patients living throughout $\mathrm{BC}$ were recruited to the patient advisory through expression of interest calls posted by the BC-PHCRN, through Patient Voices Network (a network of patients and caregivers interested in engagement opportunities with healthcare partners in BC), and through a patient engagement workshop at the Centre for Health Services and Policy Research annual conference (Vancouver, BC, March 2017). We included the following text in the invitation: 'Priority will be given to ensuring that patient partners are inclusive of the diverse communities and individuals who access primary health care services. Please let us know if and how your participation might add to diversity.' We used this information to purposively recruit patients, aiming to maximise diversity with respect to healthcare needs, regional health authority, ethnicity (including First Nations), age and gender. We also sought to include both patients with and without previous research experience. As is consistent with Canada's Strategy for Patient-Oriented Research, ${ }^{20}$ the patient advisory members were research partners 
1a. Exploration (part 1)

2. Consultation (Interim) Prioritisation

Priority setting workshop

$\checkmark$

1b. Exploration (part 2)

3. Prioritisation

4. Integration

5. Programming and implementation

Dissemination of research

Figure 1 Flow chart of key activities of the PREFeR Project, framed within the five phases of a modified dialogue model. PREFeR, PRioritiEs For Research.

in PREFeR. They contributed to planning and shaping research activities other than priority setting, since they provided input as participants to the priority setting phase of this project, as described below.

\section{Overview of the dialogue model}

Compared with the other structured priority setting frameworks, the five phases of the dialogue modelexploration, consultation, prioritisation, integration and programming and integration ${ }^{115}$ - mapped most closely on to the overarching ethos of PREFeR (figure 1). In particular, the dialogue model is based on participatory and interactive approaches, which advocate an equal partnership between patients and other stakeholders. ${ }^{1}$ Through processes that encourage mutual learning, the dialogue model can lead to co-ownership of the priorities identified. ${ }^{1}$ The model uses a variety of mixed methods (eg, literature reviews, interviews, surveys) throughout the five phases, which we also identified as essential to the aims of PREFeR. Commonly, these methods are grounded in other structured techniques. For example, focus groups may be framed within the nominal group technique (NGT), ${ }^{23}$ which is a widely used method of idea generation, based on four key stages: individual and silent idea generation in response to the nominal question, round-robin feedback to the group (ie, each person sequentially shares one idea at a time), group discussion and clarification of ideas, individual voting (ranking or rating of ideas). ${ }^{24}$

The five phases of the dialogue model were modified to match the aims of PREFeR. Most notably, we chiefly

focused on patient views in the consultation phase, since the goal of PREFeR was to generate patient priorities. However, priorities from our other stakeholders were gathered and compared with patient priorities later in the project. Below, we overview all activities in PREFeR as they map on to the phases of the dialogue model, but the primary focus of this paper is on the exploration, consultation and prioritisation phases. Research activities in these phases correspond to use of NGT to generate and prioritise patient-generated primary care topics, and province-wide online surveys to rate the importance of the top 10 patient-ranked topics among a wider group of patients and primary care providers.

\section{Data collection}

\section{Exploration phase (part 1)}

\section{Brainstorming primary care experiences}

In the first activity of the project (within the exploration phase), the 10-member patient advisory was sent an email request to brainstorm their ideas in response to the following question, 'Based on your experiences of primary care, what things really stand out for you?' Primary care was defined as where most people go first for medical help and advice when they have a health problem, and provided by healthcare professionals, like family doctors, nurses or nurse practitioners, pharmacists or emergency services staff. The question was neutrally framed by design, such that either positive or negative experiences of primary care might emerge. Patient advisory members were asked to spend about 10 minutes on the task and write down as many things that came to mind in response to this question. They were invited to a teleconference to share their ideas with the group approximately two weeks later. For those unable to attend, an opportunity to provide their thoughts through individual telephone calls with the first author prior to the scheduled teleconference was offered. With permission, these members' primary care experiences were shared with the rest of the patient advisory during the group teleconference.

The teleconference was led by the first author and facilitated by the second author. The first author has a PhD in psychology and over eightyears experience working within large, multistakeholder primary care research teams that all included patient and public involvement members. She joined the research team after the patient advisory had been recruited, did not know any of the members prior to working on the project, and the teleconference was her first interaction with the patient advisory. The first and second author worked together to ensure equal opportunity of patient voices throughout the teleconference.

Using $\mathrm{NGT}^{23}$ implemented with a teleconference platform, ${ }^{25}$ each patient advisory member was asked to provide one idea at a time, in round-robin fashion, until there were no more new ideas to share. In line with NGT, patients were asked to first briefly describe the ideas that they had in response to the question, 'what things really stand out for you in primary care?' Further explanation 
and discussion by the entire group followed after all ideas had been listed. The last author took detailed notes throughout the teleconference, which were emailed to the patient advisory the following day for their review. No identifiers were used in the notes, since the aim was to record the raw list of ideas from the entire group.

Based on the teleconference notes, the first author consolidated the raw list of brainstormed ideas. Common elements were grouped thematically into topics. A heading for each topic was constructed with a short phrase in lay language to reflect the underlying theme of the collection of examples and experiences raised by the patients. The specific patient examples and experiences were additionally provided in bullet form below each topic heading. After an initial discussion with the second and last author, the topic list was refined and then emailed to the patient advisory. The patient advisory was asked to review the list of topics ahead of an in-person priority setting workshop, to ensure it reflected the teleconference discussion accurately.

\section{Consultation and (interim) prioritisation phases Interim priority setting workshop}

Three and a half weeks after the teleconference, a full-day, in-person priority setting workshop with the patient advisory was held in Vancouver, BC. The purpose of the workshop was, first, to review, discuss, revise and agree on the list of the patient-generated primary care topics that emerged out of the teleconference (consultation phase), and then to have the patients individually rank the top 10 most important topics ((interim) prioritisation phase). Similar to the methods used by Broerse $e t a l,{ }^{15}$ we decided in advance that 10 topics would be a manageable number to carry forward for patients and primary care providers to rate in an online survey. Collectively, this constituted the remaining steps of the NGT process, which followed from the initial stages conducted during the teleconference.

To arrive at a final, agreed on list of primary care topics, the patient advisory was asked to review the topics and consider: (1) the suitability of the topic label wording, (2) whether any topics were missed from the teleconference or new ideas had emerged since that discussion and (3) whether any additional topics should be separated out from the patient examples and/or whether two or more topics could be grouped together. These points were discussed for each topic in turn, making revisions on a projected screen in real time during the workshop.

Once the list of primary care topics was finalised, the group moved on to the second task of the workshopranking the topics in terms of their importance. The final topic list was distributed to the patient advisory, who were then asked to privately choose the 10 topics that were most important to them personally. Personal importance could also include thinking about how the issues might affect other people they were close to (eg, family, friends). A recording sheet was provided for patients to arrange their top 10 topics in terms of $1=$ mostimportant to $10=$ leastimportant. Ties were not permitted. In line with NGT methods, patients were asked to complete this task silently and on their own, and they were given as much time as needed. The first author checked the ranking sheets for completeness as they were collected.

The first author entered the patient-ranked data into an Excel spreadsheet, and then reverse scored the data, such that $10=$ mostimportant and $1=$ leastimportant. Following the steps outlined in McMillan $e t a l,{ }^{24}$ results were pooled by first creating a sum of scores for each topic, which indicated its ranked priority or strength of vote score,${ }^{26}$ and then calculating the proportion (\%) of scores ranked within the top 10 topics, which indicated relative importance. ${ }^{27}$ In order to resolve ties, a third method of tabulating the ranks involved counting the number of times each topic was ranked within the top 10 across patients, which is an indication of topic popularity. ${ }^{24}$ The data were then sorted according to their ranked priority, yielding the top 10 topics that the project would carry forward in the remaining project activities.

\section{Exploration phase (part 2): rapid literature reviews}

Rapid literature reviews were carried out for English language publications from 2008 to 2018, reporting research conducted within $\mathrm{BC}$ and Canada on each of the 10 patient-identified primary care topics. This information was used to inform the Integration phase and future development of research questions, but we do not report results herein. This reflects a divergence from the dialogue model, in which we carried out rapid literature reviews after the consultation phase, instead of within the exploration phase. This modification was made to provide input to contextualise what research had already been conducted within Canada. Within the dialogue model, literature reviews may be undertaken for scoping purposes, and to directly shape subsequent activities within the model, such as we chose to do.

\section{Prioritisation phase}

Topic importance surveys

Online surveys were administered province-wide to capture BC patients' and primary care providers' importance ratings of the top 10 topics. Both stakeholder groups were asked to rate each topic individually from $1=$ not at all important to $5=$ veryimportant. The topic heading, along with 2-4 bullet points of examples and experiences (from the patient advisory) to illustrate the topic, were included to mitigate misunderstanding and misinterpretation of the topic heading. Participants were asked to rate rather than rank the identified topics, so that we could understand which topics were closely clustered with respect to importance, and not just overall rank order. A free-text box was provided for any comments respondents wished to submit. Following the importance ratings, several sociodemographic questions were included in the surveys (online supplementary file $1)$. Completion of the online survey implied consent, which was outlined on the information landing page for the survey. 


\section{Survey participants and recruitment}

We recruited survey respondents using multiple online dissemination channels, such as social media (Twitter, Facebook), e-newsletters, emails, snowballing and notices posted on several websites. We also included a one-time classified ad in 31 local newspapers in small BC communities and offered telephone completion as an alternative to the online survey. Due to time and budget constraints, the surveys were only provided in English. The sole inclusion criterion was that patients and providers must be living in $\mathrm{BC}$ to complete the survey.

\section{Analysis of survey data}

We report means (M) and standard devistions (SD) of importance ratings among patients and providers. Future analyses will explore differences in the ratings by patient and provider characteristics.

\section{Horizon scanning survey}

A third survey with BC researchers was included for horizon scanning purposes; namely, to capture any past, ongoing or upcoming (in the next 12 months) research on the 10 primary care topics. This complements the rapid literature reviews, but it also enabled us to capture research that had not been published, completed, or for which funding ha recently been secured. As with the literature reviews, this information was used to inform the Integration phase and future development of research questions, but we do not report results herein.

\section{Integration phase}

Patient, provider, researcher and policy-maker dialogue event

A final dialogue event brought patients and primary care providers together to share the survey results, and to engage in a facilitated discussion about areas of concordance and dissimilarity in the results. Patients included the patient advisory and others recruited to enhance the representation of minority populations (eg, Indigenous peoples and newcomers to the province). We recruited a diverse mix of primary care providers, including general practitioners, nurses, nurse practitioners, and pharmacists.

The aim of the event was to gain a better qualitative understanding of the general perspectives of patients and primary care providers with respect to the quantitative survey results, rather than to achieve consensus on the top primary care topics for the two stakeholder groups, as in the dialogue model. We think it is more important to understand where and how patient and provider perspectives might differ, rather than try to bring the views of a small subgroup of patients and primary care providers together.

A parallel discussion was held with researchers and policy-makers at the dialogue event. These stakeholders also discussed the survey findings, but the focus was on how these results might be translated into future research and their policy implications. Findings from this event will be reported elsewhere.

\section{RESULTS}

\section{Patient advisory characteristics}

In total, 11 members (eight female, three male) were recruited to the patient advisory between March and May 2017. Of these, 10 were recruited through recruitment advertisements placed with Patient Voices Network BC. A total of three males and 12 females replied to these ads, and we chose three males and seven females. An eighth female member was recruited from another study to ensure equal representation across the five regional health authorities in the province. However, one female patient resigned at the start of the PREFeR Project and did not provide any input to the project. The final 10 patient advisory members (seven female, three male) were characterised by a mix of sociodemographic characteristics, including ethnic minority backgrounds, speaking multiple languages, born outside of Canada or the province, and representation from all five health authorities (table 1). In line with the recruitment strategy, patients included people with both visible/congenital (one member) and invisible disabilities, experiences of long-standing chronic illness (including mental health disorders, chronic infectious disease, chronic pain). Some had recovered from very severe, life-threatening health conditions (eg, cancer), while others had only minor needs for episodic care. The group were predominantly employed (7/10, 70\%), well educated (BSc, BA, teacher's college or higher university degree: $6 / 9,67 \%$ ), and were considered 'experienced' (eg, members of three or more research committees/organisations, patient partners in other studies, research participant involvement, etc) in terms of previous research involvement $(6 / 9,67 \%)$. The group did not know each other before joining the patient advisory, and the PREFeR project was the first task the advisory worked on collectively.

\section{Brainstorming primary care experiences}

Two (one male, one female) patient advisory members were unable to attend the primary care experiences teleconference in September 2017, and so their ideas were shared via a telephone call with the first author in advance. In total, these and the remaining eight patient advisory members (two male, six female) provided 82 experiences and examples of what stood out for them in primary care in BC. There was considerable overlap in these primary care experiences, which were initially grouped into 19 themes by LE, MM and MRL (online supplementary file 2, column 1).

\section{Interim priority setting workshop}

Nine (two male, seven female) of the 10 patient advisory members attended the in-person priority setting workshop in Vancouver, Canada in September 2017. The researcher-proposed 19 primary care themes were reviewed, discussed and revised (online supplementary file 2, column 2; topic headings and full set of illustrative bullet points are also provided in online supplementary file 2). No additional themes were added, but 14 of the 


\begin{tabular}{lc}
\hline $\begin{array}{l}\text { Table } 1 \\
\text { Health Care Research Network Patient Advisory }(\mathrm{n}=10)\end{array}$ \\
\hline \multicolumn{2}{l}{$\mathbf{N}(\%)$} \\
\hline Age in years (M, SD) & $48.4(13.9)$ \\
\hline Age range in years & $24-67$ \\
Sex & \\
\hline Female & $7(70)$ \\
\hline Male & $3(30)$ \\
\hline Ethnicity & \\
\hline White only & $3(33)$ \\
\hline Indigenous & $2(20)$ \\
\hline Any other background & $4(40)$ \\
\hline Language(s) spoken ${ }^{\star}$ & \\
\hline English only & $5(56)$ \\
\hline Multiple languages & $4(44)$ \\
\hline Employed & $7(70)$ \\
\hline Yes & $3(30)$ \\
\hline No &
\end{tabular}

Highest education level ${ }^{\star}$

$\begin{array}{ll}\begin{array}{l}\text { Trade or non-university certificate/diploma, } \\ \text { community college, university certificate below BA }\end{array} & 3(33) \\ \text { BA, teacher's college or higher } & 6(67) \\ \text { Country of birth* } & \\ \text { Canada } & 8(89) \\ \text { Outside Canada } & 1(11)\end{array}$

\begin{tabular}{|c|c|}
\hline Outside Canada & $1(11)$ \\
\hline \multicolumn{2}{|l|}{ Years living in $\mathrm{BC}^{*}$} \\
\hline $0-5$ & $2(22)$ \\
\hline $6-15$ & $1(11)$ \\
\hline More than 15 years & $6(67)$ \\
\hline \multicolumn{2}{|l|}{ Regional health authority $†$} \\
\hline Vancouver Coastal Health & $5(50)$ \\
\hline Vancouver Island Health & $2(20)$ \\
\hline Fraser Health & $1(10)$ \\
\hline Interior Health & $1(10)$ \\
\hline Northern Health & $1(10)$ \\
\hline \multicolumn{2}{|c|}{ Previous research involvement ${ }^{\star} \ddagger$} \\
\hline None & $1(11)$ \\
\hline Moderate & $2(22)$ \\
\hline Experienced & $6(67)$ \\
\hline
\end{tabular}

All values reported are $\mathrm{N}(\%)$, unless otherwise specified. *Data available for 9 of the 10 patient advisory members. †There are five regional health authorities in BC. ¥Moderate $=2-3$ years of researcher/research participant experience and/or patient partner on $>2$ committees/studies; experienced $\geq 3$ years of researcher/research participant experience and/or patient partner on $>3$ committees/studies.

topic headings were changed and two of the researcher-suggested topics (impact of a new diagnosis, care of chronic conditions) were combined to form a single patient-revised topic (support for living with chronic conditions). In total, 18 final topics were retained from the first part of the workshop.

Patients selected and then ranked the 10 most important topics for themselves and in consideration of their social network. The raw rankings were followed by methods of tabulating the final ranked priority topics (via scores and votes), which included the sum of scores across patients, ranked priority via scores, relative importance, ranked priority via relative importance, and the number of votes (online supplementary file 3). There were some tied ranks, which were resolved by the number of votes. For example, accessing care when and where patients need it and support for living with chronic conditions were tied for fourth place, but the access-related topic received more votes overall, and so retained the higher priority position. There was consensus within the team that the top 10 topics captured the most important ideas.

\section{Survey participant characteristics}

Between February and May 2018, 464 patients and 173 primary care providers living and working in BC completed the survey. As shown in table 2, the majority of both patients and providers were female and of white ethnicity. The proportion of patient survey respondents from the five regional health authorities was closely aligned with 2017 provincial population estimates for Vancouver Coastal Health and Vancouver Island Health (provincial estimates: $24.6 \%$ and $16.5 \%$, respectively), but there was over-representation from Interior and Northern Health (provincial estimates: $15.7 \%$ and $5.8 \%$, respectively) and under-representation from Fraser Health (provincial estimate: $37.5 \%){ }^{28}$

Primary care providers from Interior Health authority were over-represented, constituting over half of all respondents. Providers were fairly evenly distributed between primary care physicians, nurses, and all other providers such as pharmacists, physical and occupational therapists, and social workers.

\section{Survey topic importance ratings}

Comparing between patient and provider topic importance mean ratings, all 10 topics were rated highly by both groups (between $4=$ 'important' and $5=$ 'very important') (table 3). Among both patients and providers, the top-ranked topic was unable to find a regular family doctor or other primary healthcare provider. Among patients, the next six topics (support for chronic conditions, information sharing (including electronic medical record (EMR)), mental health resources, access to care when/where needed, new models of healthcare and improve continuity/coordination) were all similarly rated (means 4.41-4.52). The same six mid-ranked topics were similarly clustered for providers, although there was a wider range of average ratings (means 4.27-4.53). The final three lower ranked topics were still equivalent to the 'important' category, on average. Therefore, there were more similarities than differences between the rank ordering of topics for patients and providers. 
Table 2 Characteristics of patients and primary care providers completing the online survey

\begin{tabular}{|c|c|c|}
\hline & $\begin{array}{l}\text { Patients } \\
(\mathrm{n}=464)^{\star}\end{array}$ & $\begin{array}{l}\text { Providers } \\
(\mathrm{n}=173)^{\star}\end{array}$ \\
\hline \multicolumn{3}{|l|}{ Age } \\
\hline Under 40years & $123(26.5)$ & - \\
\hline 40-59years & $158(34.0)$ & - \\
\hline $60+$ years & $152(32.7)$ & - \\
\hline \multicolumn{3}{|l|}{ Sex } \\
\hline Female & 368 (79.3) & $130(75.6)$ \\
\hline Male & 77 (16.6) & $35(20.4)$ \\
\hline \multicolumn{3}{|l|}{ Ethnicity } \\
\hline White only & 366 (78.9) & $130(75.1)$ \\
\hline $\begin{array}{l}\text { Mixed or any other } \\
\text { background }\end{array}$ & $74(15.9)$ & $22(12.7)$ \\
\hline \multicolumn{3}{|l|}{ Regional health authority $†$} \\
\hline Vancouver Coastal Health & $108(23.3)$ & $23(13.3)$ \\
\hline Fraser Health & 97 (20.9) & $19(11.0)$ \\
\hline Vancouver Island Health & $70(15.1)$ & $16(9.3)$ \\
\hline Interior Health & $129(27.8)$ & $99(57.2)$ \\
\hline Northern Health & $47(10.1)$ & $5(2.9)$ \\
\hline $\begin{array}{l}\text { Provincial Health Services } \\
\text { Authority }\end{array}$ & & $9(5.2)$ \\
\hline \multicolumn{3}{|l|}{ Type of provider } \\
\hline Family doctor & - & $52(30.1)$ \\
\hline Nurse & - & $52(30.1)$ \\
\hline Other primary care provider $\ddagger$ & - & $10(5.8)$ \\
\hline $\begin{array}{l}\text { Community-based/allied } \\
\text { workers§ }\end{array}$ & - & $38(22.0)$ \\
\hline $\begin{array}{l}\text { Hospital-based only, including } \\
\text { consultants }\end{array}$ & - & $21(12.1)$ \\
\hline
\end{tabular}

All values reported are $\mathrm{N}(\%)$.

*Figures do not always sum to 464 and $173(100 \%)$, due to no response/system missing, 'prefer not to answer', 'don't know' or 'other' responses.

†There are five regional health authorities in British Columbia. ¥Other primary care providers included pharmacists, midwives or any other primary care provider other than family doctors and nurses.

$\S$ Community-based/allied workers included social workers, care aides.

\section{DISCUSSION}

Involving patients in setting priorities for primary care research is feasible, fruitful and important to patients, but it is rare. This paper outlines the key activities of the PREFeR Project, which are framed within the dialogue model, and reports the processes and results from the priority setting exercises. A group of 10 patients from all five regional health authorities in the province were recruited as patient partners to a patient advisory group. Using the NGT, over 80 experiences of what stood out for them in primary care were generated. After grouping these into 18 common themes, the top 10 ranked topics were retained for rating in a province-wide survey for patients and primary care providers. The most highly rated topics for both patients and providers were about being unable to find regular family doctor/other primary healthcare provider, support for living with chronic conditions, information sharing, including EMR and mental health resources. In fact, the mean ratings and rank ordering of the topics were similar for both stakeholder groups.

The 10 priorities originally identified by the patient advisory align fairly well with some recent priority setting initiatives in the province. The BC Ministry of Health recently identified eight service delivery priorities for the province's health system in $2014 .^{29}$ These were reconfirmed at the primary care level by the BC-PHCRN research, clinical and policy leads in $2015,{ }^{21}$ but no patients were involved in this process. There is considerable overlap between the previous BC-PHCRN priorities and those reported herein, although there were two uniquely identified patient advisory priorities that were not advanced by either the BC Ministry of Health or BC-PHCRN: Mental health resources (although, access to specialist treatment, in general, was mentioned by both organisations, and reducing demand on hospitals by improving care for those with mental health and substance use issues was one of five strategic priorities for the BC Ministry of Health in $2017^{30}$ ) and improve and strengthen patientprovider communication. There were also examples where patient perspectives added depth and context. While both sets of priorities spoke to the need to address challenges specific to rural and remote settings, patients highlighted that transportation needs to be considered, and privacy and stigma may impact those in rural areas. The previous BC-PHCRN priorities included the need to enhance access, utilisation and continuity of patient information with other providers and levels of care, but patients spoke more directly to the need for electronic health records and the ability to access their medical records themselves. Finally, while the previous priorities noted issues around workforce planning, patients framed this as being unable to find a regular doctor or other primary healthcare provider. This highlights the value of including patients directly in setting priorities for primary care, and is another demonstration of how the priorities of patients and other stakeholders might differ.

The dialogue model, coupled with NGT, was a useful framework for identifying patient priorities for primary healthcare research. This model has been used for agenda setting in many condition-specific populations, such as burns, diabetes, kidney disease, cystic fibrosis, dementia, bipolar disorder, ${ }^{1}$ but PREFeR is the first instance of primary care priority setting with a generic population that we are aware of. That said, the five phases of the dialogue model were modified substantially to address the aim of PREFeR, which explicitly focuses on patient priorities. Consistent with Canada's Strategy for patient-oriented research, the patient advisory members were research partners in PREFeR and contributed to 
Table 3 Mean (SD) importance ratings of primary care topics listed in order of mean patient ratings

\begin{tabular}{lll}
\hline Primary care topics & Patients $\mathbf{( n = 4 6 4 )}$ & Providers $(\mathbf{n = 1 7 3 )}$ \\
\hline (1) Unable to find regular family doctor/other primary healthcare provider & $4.64(0.76)$ & $4.59(0.76)$ \\
(2) Support for living with chronic conditions & $4.52(0.75)$ & $4.36(0.88)$ \\
(3) Information sharing, including EMR & $4.52(0.76)$ & $4.29(0.91)$ \\
(4) Mental health resources & $4.51(0.81)$ & $4.53(0.77)$ \\
(5) Accessing care when and where patients need it & $4.46(0.78)$ & $4.27(0.85)$ \\
(6) New models of primary care that include other HCPs & $4.44(0.83)$ & $4.30(1.03)$ \\
(7) Improving continuity and coordination & $4.41(0.81)$ & $4.31(0.85)$ \\
(8) Improve and strengthen patient-provider communication & $4.21(0.92)$ & $4.03(1.05)$ \\
(9) Challenges in small towns/cities and remote areas & $4.18(0.99)$ & $4.18(0.92)$ \\
(10) Care guided by patient needs, values, preferences and priorities & $4.09(1.02)$ & $3.95(1.07)$
\end{tabular}

The 10 topics were each rated on a scale from 1 (not at all important) to 5 (very important). EMR, electronic medical record; HCPs, healthcare professionals.

planning and shaping the research activities. Their dual role as research participants and team members means that, to some degree, we shifted control and power to patients within the process, which is inconsistent with the dialogue model. This was implemented in a context where policy-makers, clinicians and researchers had previously worked to identify priorities in the absence of patients. Perspectives from these other stakeholder groups were, however, gathered and compared with patient priorities, although later in the project. In the end, these aligned quite closely, though patients identified some additional priorities and added additional context and depth to existing ones. In addition, the language used in the final topics was agreed on by patient team members, although different terminology may be more common among researchers.

Another notable divergence is that we identified topics for future research, rather than specific research questions. The top-rated topics are broad, and many reflect priorities for health system change that may be informed by research, but are not themselves research questions. Results from rapid literature reviews of the 10 topics, along with results from the 'horizon scanning' survey with $\mathrm{BC}$ researchers relating to completed, current or upcoming research on the 10 topics, will provide valuable information about the nature and extent of research on the topics. In some cases, the topics may suggest a need for strengthened knowledge translation or implementation science to inform changes to policies or service delivery rather than additional research. Also, as the searches covered broad topics, but were limited to BC and Canada, we recommend that more thorough reviews should be conducted that are specific to any future research objectives.

The main strengths of the current research are the contribution and partnership of patients in the priority setting exercises, using a structured framework to shape the research activities, and the volume of patients and providers completing the online surveys. However, there are several notable limitations to our approach. Although the initial priorities were developed by a group of patients purposively recruited to maximise diversity with regard to gender, ethnicity, prior research experience, health region and health issues, they were mostly well educated and had previous experience with research, and we cannot claim to have reached saturation in possible topics. We also decided to limit the subsequent survey to 10 topics in order to make the online surveys more manageable. We did not directly solicit additional topics from survey respondents, and there are undoubtedly topics of high priority to patients missed in this process. Given the survey was conducted predominantly online and largely distributed within networks related to healthcare and health research, respondents are unlikely to be representative of the province as a whole. This might affect the generalisability of these results.

This project demonstrates the feasibility of including patients in priority setting exercises and offers an approach that is workable within the context of primary care, rather than a condition-specific population. The identified topics overlap with, but expand on, previously identified priorities, and the patient and primary care provider surveys highlighted more alignment than divergence between these two stakeholder groups in rating the topics. Nevertheless, two novel topics addressing mental health resources and patient-provider communication were identified here, underscoring the importance of including patients in primary care priority setting. These results, coupled with those of the remaining activities in this project, will jointly provide key considerations for setting future primary care research questions.

\section{Author affiliations}

${ }^{1}$ Faculty of Health Sciences, Simon Fraser University, Burnaby, British Columbia, Canada

${ }^{2}$ Centre for Clinical Epidemiology and Evaluation, Vancouver, British Columbia, Canada

${ }^{3}$ Fraser Health Authority, Surrey, British Columbia, Canada

${ }^{4}$ BC Primary Health Care Research Network Patient Advisory, Vancouver, British Columbia, Canada

${ }^{5}$ BC SUPPORT Unit, Vancouver, British Columbia, Canada 
Acknowledgements We are grateful to the $10 \mathrm{BC}$-PHCRN Patient Advisory members (YB, Regina Cid, Chad Dickie, Penny Henderson, Brenda Jagroop, RJ, $\mathrm{KCL}$, Marilyn Parker, HP and one other who wished to remain anonymous) for volunteering their time, openly sharing their experiences and contributing so valuably to PREFeR. We thank the BC SUPPORT Unit for in-kind support and helping to publicise this study. The Patient Voices Network assisted with recruiting the patient advisory. The BC-PHCRN generously provided support to PREFeR, both administratively and through engagement with the patient advisory. The Centre for Health Services and Policy Research provided in-kind support through salary for a project coordinator. Additional coinvestigators who contributed to this project: Sabrina T. Wong, Kim McGrail, Rita McCracken, Vanessa Brcic and Victoria Schuckel.

Contributors LE and MRL designed and led all phases of the project. Specific to the results reported in this paper, LE facilitated the priority setting exercises, thematically grouped the primary care experiences into topics, carried out the priority setting data entry and rankings and drafted this paper. MRL is the PI for the PREFeR Project, oversaw all phases of the project and contributed feedback to this paper. MRL and MM recruited the patient advisory members. MM led early engagement with the patient advisory, oversaw all administrative activities for and helped facilitate the priority setting exercises and provided feedback on this paper. YB, RJ, KCL and HP are members of the BC Primary Health Care Research Network Patient Advisory and patient partners in PREFeR who provided feedback on this paper, as well as contributing to all aspects of the priority setting exercises reported herein. CM provided background information and training on research to the patient advisory, provided additional support at the priority setting workshop and commented on this paper.

Funding This work was supported by the Canadian Institutes of Health Research Patient-Oriented Research Collaboration (Grant number 151801).

Competing interests None declared.

Patient consent for publication Not required.

Ethics approval Ethics approval advice was sought and was deemed not required by the Simon Fraser University Research Ethics Board for the Consultation and Exploration phases of the project involving the Patient Advisory. Since research team members belonged to two different institutions, harmonised ethical approval was jointly granted by the Simon Fraser University and the University of British Columbia Research Ethics Boards for the topic importance surveys (Reference number 20018s0051).

Provenance and peer review Not commissioned; externally peer reviewed.

Data sharing statement № additional data are available for this study.

Open access This is an open access article distributed in accordance with the Creative Commons Attribution Non Commercial (CC BY-NC 4.0) license, which permits others to distribute, remix, adapt, build upon this work non-commercially, and license their derivative works on different terms, provided the original work is properly cited, appropriate credit is given, any changes made indicated, and the use is non-commercial. See: http://creativecommons.org/licenses/by-nc/4.0/.

\section{REFERENCES}

1. Raeymaekers P. Mind the gap! Multi-stakeholder dialogue for priority setting in health research. Brussels: King Baudouin Foundation, 2016:7-42. https://www.kbs-frb.be/en/Activities/Publications/2016/ 20160426PP (Accessed 3 Aug 2017).

2. Stewart RJ, Caird J, Oliver K, et al. Patients' and clinicians' research priorities: patients' and clinicians' research priorities. Health Expect 2011;14:439-48.

3. Tong A, Crowe S, Chando S, et al. Research priorities in ckd: report of a national workshop conducted in Australia. Am J Kidney Dis 2015;66:212-22.

4. Crowe S, Fenton M, Hall M, et al. Patients', clinicians' and the research communities' priorities for treatment research: there is an important mismatch. Res Involv Engagem 2015;1:2

5. Chalmers I, Bracken MB, Djulbegovic B, et al. How to increase value and reduce waste when research priorities are set. Lancet 2014;383:156-65.

6. Manafò E, Petermann L, Vandall-Walker V, et al. Patient and public engagement in priority setting: A systematic rapid review of the literature. PLoS One 2018;13:e0193579.

7. Kirwan JR, de Wit M, Frank L, et al. Emerging guidelines for patient engagement in research. Value Health 2017;20:481-6.
8. NAPCRG's Research Advocacy Committee. Primary care research priorities identified and shared with stakeholders. Ann Fam Med 2014;12:381-2.

9. Ball L, Barnes K, Leveritt M, et al. Developing research priorities in Australian primary health care: a focus on nutrition and physical activity. Aust J Prim Health 2017;23:554-9.

10. MacFarlane A, Galvin R, O'Sullivan M, et al. Participatory methods for research prioritization in primary care: an analysis of the World Café approach in Ireland and the USA. Fam Pract 2017;34:278-84.

11. Blaum C, Tinetti M, Rich MW, et al. A research agenda to support patient priorities care for adults with multiple chronic conditions [White paper]: Patient-Centered Outcomes Research Institute, 2017. https://www.pcori.org/sites/default/files/NYU2870-White-Paper.pdf. (Accessed 19 Jan 2019).

12. Morris RL, Stocks SJ, Alam R, et al. Identifying primary care patient safety research priorities in the UK: a James Lind Alliance Priority Setting Partnership. BMJ Open 2018;8:e020870.

13. Yoshida S. Approaches, tools and methods used for setting priorities in health research in the 21(st) century. J Glob Health 2016;6:010507.

14. The James Lind Alliance. The James Lind Alliance guidebook, Version 7. Southampton: National Institute for Health Research Evaluation, Trials and Studies Coordinating Centre, 2018. http://www. jla.nihr.ac.uk/jla-guidebook/downloads/Print-JLA-guidebook-version7-March-2018.pdf. (Accessed 16 Jul 2018).

15. Broerse JE, Zweekhorst MB, van Rensen AJ, et al. Involving burn survivors in agenda setting on burn research: an added value? Burns 2010;36:217-31.

16. The James Lind Alliance. The James Lind Alliance guidebook, Version 6. Southampton: National Institute for Health Research Evaluation, Trials and Studies Coordinating Centre, 2016. http://www. jla.nihr.ac.uk/jla-guidebook/downloads/JLA-Guidebook-Version-6February-2016.pdf. (Accessed 30 Aug 2017).

17. Ryan M, Scott DA, Reeves C, et al. Eliciting public preferences for healthcare: a systematic review of techniques. Health Technol Assess 2001;5:1-186.

18. Rudan I, Kapiriri L, Tomlinson M, et al. Evidence-based priority setting for health care and research: tools to support policy in maternal, neonatal, and child health in Africa. PLoS Med 2010;7:e1000308.

19. Cochrane Priority Setting Methods Group. Plain language summaries of research priority setting methods. https://methods.cochrane.org/ prioritysetting/plain-language-summaries-research-priority-settingmethods (Accessed 16 Jul 2018).

20. Canadian Institutes of Health Research. Canada's strategy for patient-oriented research: improving health outcomes through evidence-informed care. 2011 http://www.cihr-irsc.gc.ca/e/ documents/P-O_Research_Strategy-eng.pdf (Accessed 19 Jan 2019).

21. BC Primary Health Care Research Network. Research priorities. https://spor-bcphcrn.ca/about/research-priorities/ (Accessed $16 \mathrm{Jul}$ 2018).

22. Domecq JP, Prutsky G, Elraiyah T, et al. Patient engagement in research: a systematic review. BMC Health Serv Res 2014;14:89.

23. Delbecq A, van de Ven A, Gustafson D. Group techniques for program planning, a guide to nominal group and Delphi processes. Glenview, IL: Scott, Foresman and Company, 1975.

24. McMillan SS, Kelly F, Sav A, et al. Using the nominal group technique: how to analyse across multiple groups. Health Services and Outcomes Research Methodology 2014;14:92-108.

25. Rice DB, Cañedo-Ayala M, Turner KA, et al. Use of the nominal group technique to identify stakeholder priorities and inform survey development: an example with informal caregivers of people with scleroderma. BMJ Open 2018;8:e019726.

26. Sink DS. Using the nominal group technique effectively. Natl Product Rev 1983;2:173-84.

27. Gastelurrutia MA, Benrimoj SI, Castrillon CC, et al. Facilitators for practice change in Spanish community pharmacy. Pharm World Sci 2009;31:32-9.

28. Government of British Columbia. Sub-provincial population estimates. http://www.bcstats.gov.bc.ca/apps/PopulationEstimates. aspx (Accessed 22 Aug 2018).

29. British Columbia Ministry of Health. Setting priorities for the B.C. health system. 2014 http://www.health.gov.bc.ca/library/ publications/year/2014/Setting-priorities-BC-Health-Feb14.pdf (Accessed 16 Jul 2018).

30. British Columbia Ministry of Health. Ministry of Health patients as partners 2017 provincial dialogue report. 2017 https://www2.gov.bc. ca/assets/gov/health/about-bc-s-health-care-system/heath-carepartners/patients-as-partners/provincial-annual-dialogue-report2017.pdf (Accessed 16 Jul 2018). 